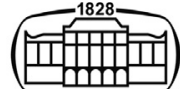

AKADÉMIAI KIADÓ

Journal of Behavioral

Addictions

$10(2021) 3,381-387$

\section{Special Section on sports and esports betting}

\section{DOI:}

$10.1556 / 2006.2020 .00054$

(c) 2020 The Author(s)

\title{
Request-a-bet sports betting products indicate patterns of bettor preference and bookmaker profits
}

\author{
PHILIP W.S. NEWALL ${ }^{1,2 *}$ (อ) LUKASZ WALASEK ${ }^{3}$, REBECCA \\ VÁZQUEZ KIESEL ${ }^{4}$, ELLIOT A. LUDVIG ${ }^{3}$ and \\ CAROLINE MEYER ${ }^{2}$
}

\author{
${ }^{1}$ Experimental Gambling Research Laboratory, School of Health, Medical and Applied Sciences, \\ CQUniversity, 120 Spencer St, 3000, Melbourne, VIC, Australia \\ ${ }^{2}$ Applied Psychology, WMG, University of Warwick, Coventry, CV4 7AL, United Kingdom \\ ${ }^{3}$ Department of Psychology, University of Warwick, Coventry, CV4 7AL, United Kingdom \\ ${ }^{4}$ Technische Universität München, Arcisstraße 21, 80333, München, Germany
}

Received: May 06, 2020 • Revised manuscript received: July 20, 2020; August 13, 2020 • Accepted: August 18, 2020

Published online: September 8, 2020

\section{FULL-LENGTH REPORT}

\begin{abstract}
Background and aims: Request-a-bet services are a modern gambling product delivered via the social network Twitter, which allow sports bettors to design custom bets. The public nature of Twitter data provided a unique opportunity to investigate patterns of bettor preference and the bookmaker profit margin in soccer, the UK's favorite sport. Methods: Two multi-method studies. Twitter users' engagement with request-a-bet services was monitored unobtrusively $(n=1,406)$, meaning that potential patterns across users' requests could be observed, and the bookmaker profit margin could be estimated. Twitter users were also surveyed directly $(n=55)$, providing self-report measures of requesta-bet usage. Results: Twitter users requested bets with an average potential payoff of $£ 56.5$ per $£ 1$ risked (median = £9). Overall, 9.7\% of requested bets paid-off, but these were mostly bets at short odds. This meant that requests yielded a high bookmaker profit margin of $43.7 \%$ (roughly eight times higher than current margins in conventional soccer bets), which increased to $74.6 \%$ for bets at longer odds. Requested bets also tended to involve star players from the best teams. Finally, $92.7 \%$ of surveyed Twitter users reported placing at least one bet via request-a-bet services (mean $=44.4$ bets). Discussion and conclusions: Researchers can use request-a-bet products to increase their understanding of sports betting behavior. Sports bettors should be given information about how much higher the bookmaker profit margin can be in modern sports bets compared to the conventional sports bets that they may be more familiar with.
\end{abstract}

\section{KEYWORDS}

modern gambling products, online gambling, sports betting, probability discounting, gambling, social media

\section{INTRODUCTION}

Modern gambling products often involve novel design features that may take advantage of disordered gamblers' biases (Schüll, 2012). For example, modern electronic gambling machines (EGMs) make it easy to bet repeatedly (Dowling, Smith, \& Thomas, 2005), thereby potentially taking advantage of deficits in impulsiveness in disordered gambling (Amlung, Vedelago, Acker, Balodis, \& MacKillop, 2017; MacKillop et al., 2011). By comparison, there has been less research on modern sports betting products, perhaps due to only recently lifted legal restrictions around US sports betting (Supreme Court of the United States, 2017). "Request-a-bet" services have recently been launched in the UK and allow gamblers to design 
custom bets via the social network Twitter. Compared to conventional sports betting products, request-a-bets allow gamblers to imagine from scratch what they would like to bet on and place a bet that perhaps no one else in the world has selected. This customization allows bettors to imagine that they can beat the odds with their own personal control, perhaps taking advantage of the illusion-of-control gambling bias (Langer, 1975).

In addition, request-a-bet products can also provide a unique perspective on patterns of bettor preference and bookmaker profits compared to conventional sports betting. Data on how sports bettors actually bet are not routinely made available to researchers (Cassidy, Loussouarn, \& Pisac, 2013; Chagas \& Gomes, 2017). Researchers have occasionally used prediction contests (Brown \& Reade, 2019; Butler, Butler, \& Eakins, 2020; Levitt, 2004) or built their own experimental betting platforms (Simmons, Nelson, Galak, \& Frederick, 2011), but these data sources may also fail to properly capture naturally-occurring sports betting behavior. By comparison, due to the public nature of request-a-bet services, it is possible for researchers to unobtrusively monitor large samples of custom bet requests, and to track patterns across the bookmaker profit margin based on the odds offered in reply.

Most research into conventional sports betting uses the odds that gambling operators post and the resultant realworld outcomes. For bets on the three main outcomes of a soccer match (home win, draw, away win, called "homedraw-away" bets here), this research initially found a constant bookmaker profit margin of $10.5 \%$ in the late $1990 \mathrm{~s}$ (Forrest \& Simmons, 2001; Kuypers, 2000). Online gambling and increased competition led to gradually declining profit margins of around $7 \%$ in the early 2010s (Constantinou \& Fenton, 2013), and then to around $5 \%$ by the late 2010 s (Buhagiar, Cortis, \& Newall, 2018). However, a common finding across these studies over time is that bets at long odds tend to have the highest bookmaker profit margins (Buhagiar et al., 2018; Cain, Law, \& Peel, 2000; Constantinou \& Fenton, 2013; Hassanniakalager \& Newall, 2019): the occasional large wins at long odds are insufficient compensation for the average string of losses required to yield a win. But because the types of bets that bettors actually choose are not normally observable, assumptions on bettor behavior are needed to calculate these profit margins (Stark \& Cortis, 2017).

Conventional home-draw-away bets also generally do not provide large potential wins due to the small number of potential outcomes. By contrast, UK gambling advertising around soccer tends to focus on bets at long odds, but which nonetheless require several events, often involving star players from the best teams, in order to payoff (Newall, 2015; Newall, Thobhani, Walasek, \& Meyer, 2019). This is relevant given that previous research in cognitive psychology suggests that the probabilities of such typical "representative" events are the most likely to be overestimated (Tversky \& Kahneman, 1983). However, it is unknown how popular these types of bets actually are, or what the bookmaker profit margin precisely is on these bets.
The aims of the present research were to take advantage of the public nature of Twitter data to investigate patterns in bettor preference and bookmaker profitability across a large sample of soccer bet requests. Although the data from request-a-bet products have unique advantages compared to conventional sports betting products, there are limitations too. Chiefly, we can observe requests, but not the number of bets that were later actually made. It is possible that Twitter users are engaging with request-a-bets as a source of fun or information, rather than as a method of betting. Therefore, we also administered a brief five-question survey to requesta-bet hashtag users to see how often they reported using these services to actually place bets. Some recent self-report data has suggested that disordered gamblers are especially likely to customize their sports bets, whether on Twitter or on slightly more restricted services on bookmakers' websites (Newall, Cassidy, Walasek, Ludvig, \& Meyer, 2020). The survey therefore also contained the three-item Problem Gambling Severity Index (PGSI) Short Form disordered gambling screener (Volberg \& Williams, 2012), in order to briefly assess the degree to which request-a-bet usage is correlated with disordered gambling.

\section{METHOD}

Several gambling companies' Twitter accounts now offer "request-a-bet" services, which respond to betting odds requests on custom bets from the public. Betting odds requests can be sent by any member of the public who has a Twitter account via a message accompanied by a hashtag. The bookmaker replies to betting odds requests with the custom bet's quoted odds and a link where the bet can be placed via their online platform (the bookmaker may also refuse to quote odds).

\section{Study 1: Twitter requests}

The leading high street (retail) British bookmaker William Hill runs an online request-a-bet service on Twitter via the hashtag \#MyOdds. We used the StreamR package (Barbera, 2015) in $\mathrm{R}$ to collect bet requests with the \#MyOdds hashtag and William Hill's reply using Twitter's official API. Data were collected between August 11th and September 27th, 2017, coinciding with the start of the 2017/2018 English Premier League soccer season. During this time period, only two British bookmakers that we were aware of, Paddy Power and William Hill, had request-a-bet products. William Hill has a much larger presence in the UK gambling market and created its product first, in January 2017 (Roarty, 2017).

After removing non-English characters and tweets containing only links, we obtained a total of 9,443 tweets. William Hill did not quote odds on 1,066 requests. We also did not find the original requests for 1,426 odds provided by William Hill. We further limited the sample to those involving at least one Premier League soccer event (the UK's highest level of soccer, and one of the most globally followed sports leagues). The final sample consisted of 2,889 requests and corresponding quoted odds from 1,406 unique usernames. 
There were many content features that could have potentially been extracted from the requests in order to explore patterns of bettor preference. We decided to focus on the identity and teams of all players requested to score a goal. This is because many gambling adverts for soccer in the UK tend to feature the odds on specific star players scoring a goal (Newall, 2015; Newall et al., 2019). Furthermore, scoring a goal is a clear skill-based measure, which occurred often in the dataset: 1,067 requests identified at least one named player $(M=1.39)$ to score at least one goal $(M=$ 1.07).

A number of exclusions were then necessary to create a subset of bets that could be used in order to estimate the bookmaker profit margin, which are summarized in Table 1. This was done in mid-2018, after the completion of the 2017/2018 soccer club season. Although detailed match statistics are freely available online, e.g., from premierleague. $\mathrm{com} /$ results, these statistics do not include information on some requested events, such as the number of throw-ins or goal-kicks. This led to the loss of 385 observations. At that point in time, events corresponding to a small number of requests had not yet occurred, leading to a loss of seven additional observations. A further 29 observations were otherwise codable, but included specific players who took no part in the match, invalidating the request at the bookmaker's quoted odds. This reduced the sample size to 2,468 requests for which average bet payoffs were calculated (85.54\% of the sample).

Because we do not observe bets or bet sizes, the bookmaker profit margin was calculated by assuming that bettors bet a constant amount $(£ 1)$ on each bet. This is a common assumption in soccer betting research (Kuypers, 2000). Any winning bets received profits based on the quoted odds from the bookmaker's reply. The bookmaker profit margin is then calculated as the average amount of money lost per-bet.

One researcher performed the manual coding. A $10 \%$ random sample was dual-coded to determine inter-coder reliability for the determination of bet payoffs. The coders were in $96.9 \%$ agreement when coding whether a bet should be excluded for one of these three reasons, and if it should be included, whether it paid off. This is above the suggested minimum threshold of $70 \%$ agreement for acceptable interrater percentage agreement (Stemler \& Tsai, 2008). Any disagreements were settled via discussion. In order to ensure

Table 1. Summary of exclusions prior to determining whether a bet's outcome could be determined

\begin{tabular}{lcc}
\hline Category & $\begin{array}{c}\text { Frequency } \\
\text { (proportion) }\end{array}$ & $\begin{array}{c}\text { Average } \\
\text { odds }\end{array}$ \\
\hline Information not available & $385(13.33 \%)$ & 8.75 \\
Events not yet occurred & $7(0.24 \%)$ & 10.86 \\
Specific player not & $29(1.00 \%)$ & 82.87 \\
$\quad$ involved & & \\
Overall not codeable & $421(14.57 \%)$ & 13.89 \\
Codeable & $2,468(85.54 \%)$ & 63.79 \\
Overall & $2,889(100 \%)$ & 56.52 \\
\hline
\end{tabular}

the anonymity of Twitter users, we are unable to share the text of the tweets, usernames, or meta-data that can be used to identify them. We are able to share the extracted odds of each request and the coding results. This information along with the coders' instructions sheet is available from https:// osf.io/a6kg9/.

\section{Study 2: Survey of Twitter users}

From December 2018 to March 2019 we collected usernames associated with any of the following seven request-abet hashtags (each associated with one leading UK gambling operator): \#pickyourpunt, \#priceitup, \#betyourway, \#buildyourbet, \#getaprice, \#requestabet, \#yourodds. Using the streamR package, 4,584 unique usernames were obtained for potential recruitment to a short online survey. All Twitter users can be contacted publicly by default, but Twitter users may also opt-in to allow anyone with a Twitter account to message them privately as well. Non-commercial user accounts who had opted-in to receiving private messages $(N=$ 779) were contacted with a link to a five-question survey.

The survey had to be extremely brief, as participants were unpaid. The survey asked participants to recall how many request-a-bet bets they had made, and whether the participant had won or lost overall at request-a-bets. The survey also contained the three-item PGSI Short Form disordered gambling screener (Volberg \& Williams, 2012), in order to briefly assess the degree to which request-a-bet engagement is correlated with disordered gambling. In total, 55 participants completed the survey. Questionnaire materials and anonymous responses are available from https:// osf.io/a6kg9/.

\section{Ethics}

The study procedures were carried out in accordance with the Declaration of Helsinki. The Institutional Review Board of the University of Warwick approved the survey. All subjects were informed about the study, and all provided informed consent. The analysis of collected tweets did not require ethical approval.

\section{RESULTS}

\section{Study 1: Twitter requests}

Most requested bets came at long odds. The types of bets requested likely did not reflect the average bettor's demand, because bookmakers already list many conventional bets on their websites which customers did not need to request via Twitter. Nonetheless, requested bets would have returned an average of $£ 56.52$ per $£ 1$ risked, if successful (based on the full sample, $N=2,889$ ). By comparison, consider the fact that, historically, conventional soccer bets could return a little under $£ 3$ on average per $£ 1$ risked, given the bookmaker profit margin (Buhagiar et al., 2018). The average potential returns of requested bets in our sample were also skewed by some extremely low-likelihood requests 
(maximum $=£ 10,001$ return per $£ 1$ risked). The median bet request would have returned $£ 9$ per $£ 1$ risked, which is still three times higher than a conventional soccer bet.

The subset of bets for which outcomes could be determined had slightly longer odds than the overall sample, with an average potential return of $£ 63.79$ per $£ 1$ risked, as summarized in Table 1 . We found that 240 of these 2,468 bets paid-off, or $9.7 \%$. We evaluated a strategy of betting $£ 1$ on every single of these 2,468 bets, with winning bets receiving the payoff implied from the bookmaker's odds. This revealed a bookmaker profit margin of $43.7 \%$, which is roughly eight times higher than the current margins in conventional home-draw-away soccer bets (Buhagiar et al., 2018).

Fig. 1 illustrates that most winning bets came at shorter odds $(M=4.79, N=240)$. Losing bets had on average longer odds $(M=70.15, N=2,228)$. The bookmaker profit margin increased for the longest-odds bets, as can be inferred from Fig. 1. Only three out of 724 requests with potential returns of $£ 30$ or more per $£ 1$ risked paid off, corresponding to an even higher bookmaker profit margin of $74.6 \%$ for this subset of bets. This pattern of longshot bets having the highest bookmaker profit margin replicates what has previously been observed in conventional soccer betting (Buhagiar et al., 2018; Cain et al., 2000; Constantinou \& Fenton, 2013; Hassanniakalager \& Newall, 2019).

Fig. 2 shows the numbers of time that a request specified an individual player to score at least one goal. Requests were dominated by players from the Premier League's "top six": the teams Manchester United, Manchester City, Tottenham, Liverpool, Arsenal and Chelsea. These six successful teams also have the largest fanbases, meaning that fans' preference to bet on their own team would predict a similar pattern of results (Massey, Simmons, \& Armor, 2011). Inspection of requested players' identities, however, showed that most teams' requests were dominated by a single high-profile player. Romelu Lukaku was the most expensive Premier League player transferred during the 2017 summer transfer window immediately prior to data collection, for $£ 76$ million from Everton to Manchester United. Lukaku was bought for the explicit reason to score goals. As an individual, Lukaku featured in more bet requests than any other team bar Manchester City. Among other most highly featured players were Harry Kane, who was the Premier League's top goalscorer for the two previous seasons, and Sergio Agüero, who would go on to break the Manchester City goalscoring record that season. However, these skilled players featured more heavily than their goalscoring records for the rest of the season would go on to indicate. The most highlyrequested player for each team was included in $75.1 \%$ of the requests mentioning their corresponding team, but would consequently go on to score only $24.3 \%$ of their team's goals for the season on average. This pattern of requests focusing on star players to score a goal mirrored a similar pattern that has previously been observed in UK soccer gambling advertising (Newall, 2015; Newall et al., 2019).

\section{Study 2: Survey of Twitter users}

From the 55 individuals who completed the survey, 51 participants indicated they had placed at least one request-abet wager, with a range from zero to 250 bets placed (median $=20, M=44.4, S D=65.5$ ). This result indicates that many gamblers are using request-a-bet services to gamble, rather than using the services purely for information or enjoyment. Of the participants who had bet, $37.3 \%$ reported being a winner on their request-a-bets. Participants scored an average of $1.4(S D=1.4)$ on the PGSI Short Form screen. There was a small positive correlation between the number of request-a-bets placed and PGSI score $(r=0.248)$, but which was not significant at the $5 \%$ level $(P=0.070)$.

\section{DISCUSSION}

This paper explored patterns in bettor preference and bookmaker profitability in request-a-bet products on Twitter. Twitter users requested bets with large potential payoffs compared to conventional soccer bets. These payoffs rarely occurred, however, meaning that these bets would have incurred a bookmaker profit margin of $43.7 \%$, increasing to $74.6 \%$ for outcomes potentially paying off $£ 30$

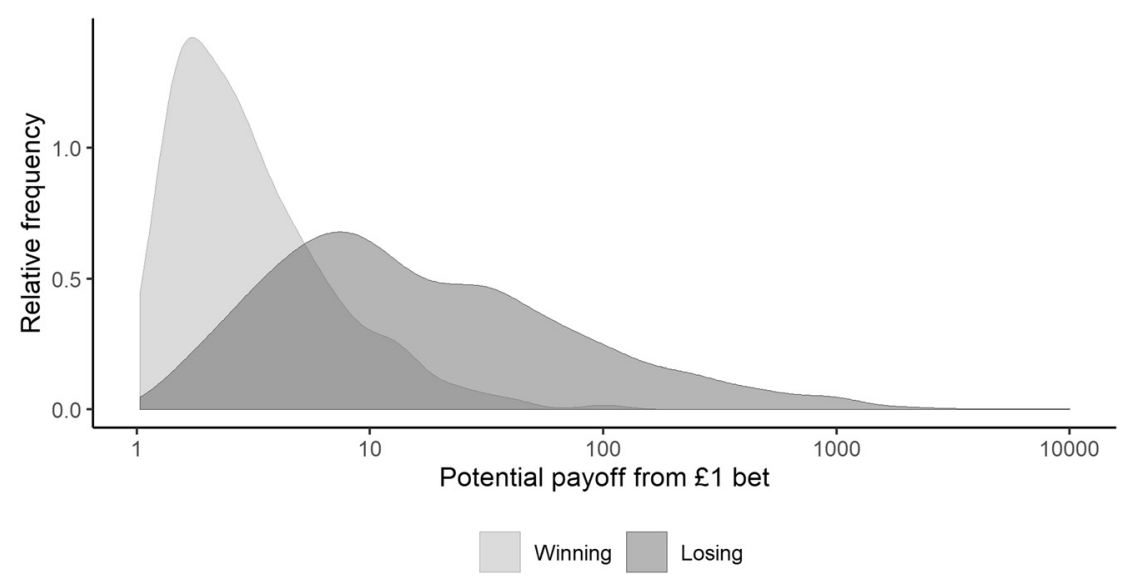

Fig. 1. Distribution of potential payoff from a $£ 1$ bet in log space for winning and losing bets 


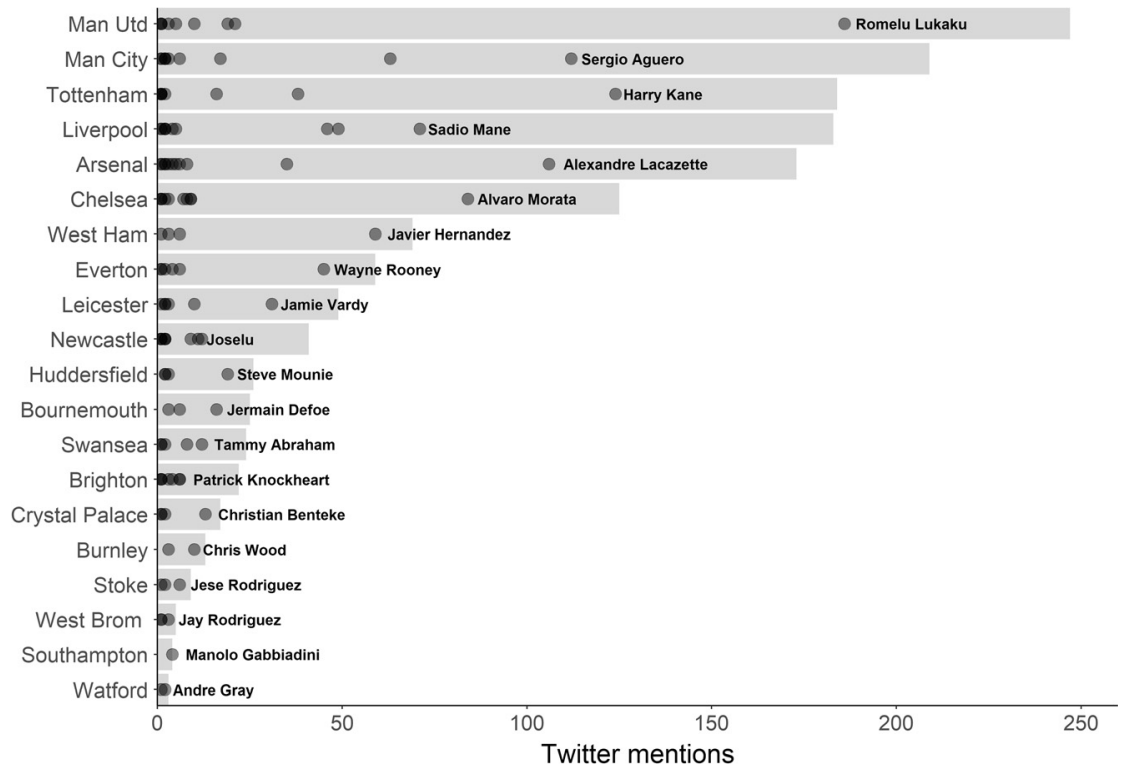

Fig. 2. Frequency distribution of individual players (dots) and their respective teams (bars) requested for all events where an individual player was required to score at least one goal. One star player dominates most teams' requests, and the name of the most frequently requested player for each team is included

or more for every $£ 1$ risked. Unlike other studies of the bookmaker profit margin in soccer (Buhagiar et al., 2018; Cain et al., 2000; Constantinou \& Fenton, 2013; Forrest \& Simmons, 2001), this study was also able to investigate patterns across the bets that people enquired about. This revealed that goalscorer requests tended to be concentrated in just a few star players from the best teams, which is consistent with the representativeness heuristic from cognitive psychology (Tversky \& Kahneman, 1983). The survey results indicated that many request-a-bet hashtag users do use these services to frequently gamble, rather than using the services purely for information or enjoyment.

It might be argued that modern sports betting products are simply helping bookmakers to better satisfy bettors' preferences. In support of this view, request-a-bet users in this study requested bets with long odds, the best teams, and star players - patterns that have been previously observed in UK gambling advertising (Newall, 2015; Newall et al., 2019). One counterargument is that UK gamblers' most commonly endorsed reason to gamble is "to win," (46\%), and their third-most commonly endorsed reason is "to win big" (19\%) (Gambling Commission, 2018). The high bookmaker profit margins observed in the present study suggest that requesta-bet products do not provide gamblers with a credible chance of winning big.

The illusion of control is a bias frequently associated with disordered gambling (Goodie \& Fortune, 2013). Although request-a-bet services appear relevant to the illusion of control, the present results do not provide evidence for any direct link between illusion of control beliefs and request-abet engagement. Similar to request-a-bet services, many bookmakers additionally offer "edit-bet" services on their websites, giving gamblers additional scope to customize their sports bets. In a previous cross-sectional survey of 789 online sports bettors, we found a statistically significant correlation $(r=0.08, P=0.031)$ between endorsement of illusion of control scale items and having ever customized a sports bet through one or more of these services (Newall et al., 2020). Although this correlation is relatively low, the illusion of control scale items were largely concerned with non-skilled gambling games such as roulette (Raylu \& Oei, 2004). The gambling field has explored cognitive illusions such as the illusion of control less in sports betting than in non-skilled gambling games (Russell, Hing, \& Browne, 2019). Further research should continue to investigate the role of the illusion of control in modern sports betting products.

There are a number of limitations to the two studies reported here. It is unknown how many of the Twitter requests were actually bet on, as is how that subsample of placed bets may differ from the overall pattern of requests observed here. This is a limitation which also afflicts the majority of studies investigating the bookmaker profit margin in sports betting (Buhagiar et al., 2018; Constantinou \& Fenton, 2013; Hassanniakalager \& Newall, 2019; Kuypers, 2000). Compared to previous research, however, the odds included in the present analysis were at least based on Twitter users' requests, rather than being based on all posted odds. The bookmaker profit margin was estimated across $85.5 \%$ of the original sample, and inclusion of the additional un-codable requests may have led to slightly different results. Overall margins may differ from these estimates, as may the effect of longshots having the highest margins (for example, if a rare event, such as Leicester winning the 2016 English Premier League, had occurred in this sample). Each study only provided a snapshot in time, and these observed patterns may change over time. Although the study of Twitter bet requests should have been representative for its 
time, it is unknown how the subset of users engaging with the survey may have differed from request-a-bet hashtag users in general. In particular, this second study was only able to contact users who had opted-in to receiving private messages, who might differ from the overall population of Twitter users. The survey did not find a statistically significant correlation between PGSI and self-reported number of request-a-bets placed, but this test had low statistical power due to the number of participants who took part in the survey. An improved study might be one that contacts request-a-bet hashtag users directly with a survey after they have requested odds on a bet, in order to determine what fraction of betting requests are bet on.

The present study showed how request-a-bet products can, unlike conventional sports betting products, reveal patterns in sports bettor behavior. By allowing customers to construct their own personal bets, request-a-bet products are likely to capitalize on the illusion of control in gambling (Langer, 1975). The pattern of requests for star players from the best teams was consistent with the representativeness heuristic from cognitive psychology (Tversky \& Kahneman, 1983). In addition, sports bettors should be given information about how much higher the bookmaker profit margin can be in modern sports bets products compared to the conventional sports bets that they may be more familiar with (Hassanniakalager \& Newall, 2019).

Funding sources: This research was funded by a Research Development Fund awarded to Lukasz Walasek from the University of Warwick.

Authors' contribution: PN and LW developed the study concept. All authors contributed to the study design. PN and RVK performed the data analysis and interpretation under the supervision of LW. PN drafted the manuscript, and LW, $\mathrm{EL}$, and $\mathrm{CM}$ provided critical revisions.

Conflict of interest: Philip Newall was a special advisor to the House of Lords Select Committee Enquiry on the Social and Economic Impact of the Gambling Industry. In the last three years Philip Newall has contributed to research projects funded by GambleAware, Gambling Research Australia, NSW Responsible Gambling Fund, and the Victorian Responsible Gambling Foundation. In 2019 Philip Newall received travel and accommodation funding from the Spanish Federation of Rehabilitated Gamblers. In 2020 Philip Newall received an open access fee grant from Gambling Research Exchange Ontario. Elliot Ludvig was coinvestigator on a grant funded by the Alberta Gambling Research Institute that ended in February 2019. The other authors have no interests to declare.

Acknowledgments: We thank Gregory Efthimiou and Ankush Thobhani for their research assistance.

\section{REFERENCES}

Amlung, M., Vedelago, L., Acker, J., Balodis, I., \& MacKillop, J. (2017). Steep delay discounting and addictive behavior: A metaanalysis of continuous associations. Addiction, 112(1), 51-62.

Barbera, P. (2015). Package 'streamR'. Retrieved from http://cran-r. c3sl.ufpr.br/web/packages/streamR/streamR.pdf.

Brown, A., \& Reade, J. J. (2019). The wisdom of amateur crowds: Evidence from an online community of sports tipsters. European Journal of Operational Research, 272(3), 1073-1081.

Buhagiar, R., Cortis, D., \& Newall, P. W. S. (2018). Why do some soccer bettors lose more money than others? Journal of Behavioral and Experimental Finance, 18(2018), 85-93. https:// doi.org/10.1016/j.jbef.2018.01.010.

Butler, D., Butler, R., \& Eakins, J. (2020). Expert performance and crowd wisdom: Evidence from English premier league predictions. European Journal of Operational Research, https://doi. org/10.1016/j.ejor.2020.05.034.

Cain, M., Law, D., \& Peel, D. (2000). The favourite-longshot bias and market efficiency in UK football betting. Scottish Journal of Political Economy, 47(1), 25-36.

Cassidy, R., Loussouarn, C., \& Pisac, A. (2013). Fair game: Producing gambling research - The goldsmiths report. London: Goldsmiths, University of London.

Chagas, B. T., \& Gomes, J. F. (2017). Internet gambling: A critical review of behavioural tracking research. Journal of Gambling Issues, 36. doi:10.4309/jgi.2017.36.1.

Constantinou, A. C., \& Fenton, N. E. (2013). Profiting from arbitrage and odds biases of the european football gambling market. The Journal of Gambling Business and Economics, 7(2), 41-70.

Dowling, N., Smith, D., \& Thomas, T. (2005). Electronic gaming machines: Are they the 'crack-cocaine'of gambling? Addiction, 100(1), 33-45.

Forrest, D., \& Simmons, R. (2001). Globalisation and efficiency in the fixed-odds soccer betting market. Unpublished manuscript.

Gambling Commission. (2018). Gambling participation in 2017: Behaviour, awareness and attitudes. Retrieved from https:// www.gamblingcommission.gov.uk/PDF/survey-data/ Gambling-participation-in-2017-behaviour-awareness-andattitudes.pdf.

Goodie, A. S., \& Fortune, E. E. (2013). Measuring cognitive distortions in pathological gambling: Review and meta-analyses. Psychology of Addictive Behaviors, 27(3), 730-743.

Hassanniakalager, A., \& Newall, P. W. S. (2019). A machine learning perspective on responsible gambling. Behavioural Public Policy, https://doi.org/10.1017/bpp.2019.9.

Kuypers, T. (2000). Information and efficiency: An empirical study of a fixed odds betting market. Applied Economics, 32(11), 1353-1363.

Langer, E. J. (1975). The illusion of control. Journal of Personality and Social Psychology, 32(2), 311-328.

Levitt, S. D. (2004). Why are gambling markets organised so differently from financial markets? The Economic Journal, 114(495), 223-246. 
MacKillop, J., Amlung, M. T., Few, L. R., Ray, L. A., Sweet, L. H., \& Munafò, M. R. (2011). Delayed reward discounting and addictive behavior: A meta-analysis. Psychopharmacology, 216(3), 305-321.

Massey, C., Simmons, J. P., \& Armor, D. A. (2011). Hope over experience: Desirability and the persistence of optimism. Psychological Science, 22(2), 274-281.

Newall, P. W. S. (2015). How bookies make your money. Judgment and Decision Making, 10(3), 225-231.

Newall, P. W. S., Cassidy, R., Walasek, L., Ludvig, E. A., \& Meyer, C. (2020). Who uses custom sports betting products? Addiction Research and Theory, 14(6), e0216876. https://doi. org/10.1080/16066359.2020.1792887.

Newall, P. W. S., Thobhani, A., Walasek, L., \& Meyer, C. (2019). Live-odds gambling advertising and consumer protection. PLoS One, https://doi.org/10.1371/journal.pone.0216876.

Raylu, N., \& Oei, T. P. (2004). The gambling related cognitions scale (GRCS): Development, confirmatory factor validation and psychometric properties. Addiction, 99(6), 757-769.

Roarty, N. (2017). William hill \#YourOdds betting markets explained we speak to william hill about their new twitter specials. Retrieved from https://www.bettingpro.com/category/opinion/william-hillyourodds-betting-markets-explained-20170123-0009/.

Russell, A. M., Hing, N., \& Browne, M. (2019). Risk factors for gambling problems specifically associated with sports betting.
Journal of Gambling Studies, 35, 1211-1228. https://doi.org/10. 1007/s10899-019-09848-x.

Schüll, N. D. (2012). Addiction by design: Machine gambling in Las Vegas. Princeton, NJ: Princeton University Press.

Simmons, J. P., Nelson, L. D., Galak, J., \& Frederick, S. (2011). Intuitive biases in choice versus estimation: Implications for the wisdom of crowds. Journal of Consumer Research, 38(1), $1-15$.

Stark, D., \& Cortis, D. (2017). Balancing the book: Is it necessary and sufficient? The Journal of Gambling Business and Economics, 11(1), 1-6.

Stemler, S. E., \& Tsai, J. (2008). Best practices in interrater reliability: Three common approaches. In J. Osborne (Ed.), Best practices in quantitative methods (pp. 29-49). CA, USA: Sage Publications.

Supreme Court of the United States. (2017). Murphy vs. national collegiate athletic association. Retrieved from https://www. supremecourt.gov/opinions/17pdf/16-476_dbfi.pdf.

Tversky, A., \& Kahneman, D. (1983). Extensional versus intuitive reasoning: The conjunction fallacy in probability judgment. Psychological Review, 90(4), 293-315.

Volberg, R., \& Williams, R. (2012). Developing a short form of the PGSI: Report to the gambling commission. Great Britain: Gemini Research. 